\title{
Combining immunotherapy with radiation therapy in thoracic oncology
}

\author{
Shahed N. Badiyan ${ }^{1}$, Michael C. Roach ${ }^{2}$, Michael D. Chuong ${ }^{3}$, Stephanie R. Rice ${ }^{1}$, Nasarachi E. \\ Onyeuku $^{1}$, Jill Remick ${ }^{1}$, Srinivas Chilukuri ${ }^{4}$, Erica Glass ${ }^{1}$, Pranshu Mohindra ${ }^{1}$, Charles B. Simone II $^{1}$ \\ ${ }^{1}$ University of Maryland School of Medicine, Baltimore, MD, USA; ${ }^{2}$ Washington University School of Medicine, St Louis, MO, USA; ${ }^{3}$ Miami \\ Cancer Institute at Baptist Health South Florida, Miami, FL, USA; ${ }^{4}$ Apollo Proton Cancer Center, Chennai, India \\ Contributions: (I) Conception and design: All authors; (II) Administrative support: None; (III) Provision of study materials or patients: None; (IV) \\ Collection and assembly of data: All authors; (V) Data analysis and interpretation: None; (VI) Manuscript writing: All authors; (VII) Final approval of \\ manuscript: All authors. \\ Correspondence to: Shahed N. Badiyan, MD. University of Maryland School of Medicine, 850 W Baltimore St, Baltimore, MD 21201, USA. \\ Email: shahedbadiyan@gmail.com.
}

\begin{abstract}
Thoracic malignancies comprise some of the most common and deadly cancers. Immunotherapies have been proven to improve survival outcomes for patients with advanced nonsmall cell lung cancer (NSCLC) and show great potential for patients with other thoracic malignancies. Radiation therapy (RT), an established and effective treatment for thoracic cancers, has acted synergistically with immunotherapies in preclinical studies. Ongoing clinical trials are exploring the clinical benefits of combining RT with immunotherapies and the optimal manner in which to deliver these complementary treatments.
\end{abstract}

Keywords: Radiation therapy (RT); immune checkpoint inhibitors (ICIs); immunotherapy; lung cancer; mesothelioma; esophageal cancer; thymoma

Submitted May 02, 2018. Accepted for publication May 08, 2018.

doi: $10.21037 /$ jtd.2018.05.73

View this article at: http://dx.doi.org/10.21037/jtd.2018.05.73

\section{Introduction}

Thoracic malignancies, consisting predominantly of nonsmall cell lung cancer (NSCLC) and small cell lung cancer (SCLC), have a predilection for distant metastasis and are associated with a poor prognosis (1). Similar outcomes are seen in other cancers of the thorax including malignant pleural mesothelioma (MPM) (2), advanced esophageal cancers (3), advanced stage thymoma, and thymic carcinoma (4). Clearly, novel therapies are needed for these patients to improve tumor control and survival.

In recent years the benefit of immunotherapy, which harnesses the body's ability to eliminate cancer cells, has emerged for some patients with thoracic malignancies. In fact, the influence of immunotherapy has been so profound that Science magazine named immunotherapy as the "Breakthrough of the Year" in 2013 (5), and it is now considered to be the fourth pillar of cancer care (6). The spotlight has focused especially on immune checkpoint inhibitors (ICIs) $(7,8)$, although other types of immunotherapies including tumor vaccines and chimeric antigen receptor (CAR) T-cell therapy are also promising $(9,10)$.

While immunotherapy alone has shown impressive results in patients with NSCLC beyond what is achievable with conventional therapies, the majority of patients will not achieve such responses. In fact, immunotherapy monotherapy is expected to result in significant benefits for only a subset of patients, especially those with high tumor mutational burden (11). Fortunately, emerging data demonstrate anti-tumor effects may increase with the use of immunotherapy in combinatorial regimens, including dual immune checkpoint blockade $(12,13)$ as well as immunotherapy plus local tumor treatment such as 
radiation therapy (RT) (14).

While radiation has direct cytotoxic effects on cancer cells, it is well established that RT is capable of generating a robust anti-tumor immune response through effects on the tumor and the tumor microenvironment via a variety of mechanisms including enhanced tumor antigen presentation and upregulated major histocompatibility complex (MHC) class I expression (15). An abundance of preclinical studies demonstrates that radiation combined with checkpoint blockade results in a synergistic effect (16). While the optimal combination in cancer patients remains unclear, there are emerging clinical outcomes data suggesting that RT may, in fact, have systemic effects that go far beyond the locally irradiated target $(17,18)$.

Herein, we review the preclinical data and clinical outcomes that support the use of radiation and immunotherapy for patients with thoracic malignancies. We also discuss ongoing clinical trials that aim to further expand our understanding of how this treatment combination may further improve the therapeutic index for these patients.

\section{Preclinical rationale for combining radiation with ICls}

\section{The tissue microenvironment (TME) of the lung}

The TME is a complex interplay of neutrophils, T-regulatory cells (Tregs), and myeloid-derived suppressor cells (MDSCs). There are pattern recognition receptors expressed by epithelial cells that can recognize and respond to pathogens through various pathways, as well as kill pathogens via these mechanisms $(19,20)$. The ratio of specific subtypes of immune cells has been shown to be associated with survival in NSCLC, with inactivated mast cells, inactivated CD4+ memory T-cells, and naïve and memory B cells and plasma cells having favorable prognosis in adenocarcinomas; whereas higher abundance of myeloid populations and activated CD4+ memory T-cells are associated with poor prognosis in squamous cell carcinomas (21). Many studies evaluating various interactions of these immune cells have been performed, and overall the tumor assisted macrophages (TAMs), neutrophils, MDSCs, and Tregs all play a role in the TME, with increased T-cell infiltration into tumor, mature dendritic cells, and B cells correlating with favorable prognosis (22).

\section{$R T$ and immune cell response}

RT has immunomodulation features within the lung parenchyma that can elicit contradictory host responses. RT can beneficially enhance recognition of tumor antigens by augmenting the immune system's response to the tumor via increased MHC class 1 expression (23). RT has also been shown to increase levels of transforming growth factorbeta (TGF- $\beta$ ), which can increase Treg representation after RT (24), resulting in a decrease in the response to therapy. RT also recruits MDSCs and macrophages, which in the presence of TGF- $\beta$ can become M2 polarized and promote tumor growth, invasion and metastasis (25). Other preclinical work focusing on the immune response of varying RT fractionation schemes shows a difference in immune cell recruitment with fractionation, noting more CD8+ recruitment in higher dose-per-fraction (30 Gy in 1 fraction) schemes compared to more MDSC recruitment with more fractionated approaches ( $3 \mathrm{~Gy} \times 10$ fractions) (26-28). As a result of the varying ways that RT can influence the immune system, clinical responses have been mixed.

\section{Rationale for the combination of ICIs and RT}

The two most actively studied immune-checkpoint receptors are both inhibitory receptors: cytotoxic T-lymphocyte-associated antigen 4 (CTLA-4) and programmed cell death protein 1 (PD-1). In general, inhibitory ligands and receptors that regulate T-cell effector functions are overexpressed on tumor cells, while costimulatory receptors that regulate T-cell activation are not overexpressed (29). One of the most common mechanisms of immune checkpoint blockade is antibodies to the PD-1 receptor. The role of PD-1 is to limit the activity of T-cells in peripheral tissues at the time of an inflammatory response to infection in order to minimize autoimmunity (30-36). However, in the TME, this process can result in effective immune resistance (37-39).

Inhibition of this checkpoint affects membrane proteins that are located on a range of cells including T-cells, B cells, natural killer (NK) cells, activated monocytes, and dendritic cells (36). Tumor cells frequently express the ligand programmed death-ligand 1 (PD-L1) on their surface, which when bound by PD-1, downregulate antigen receptor signaling resulting in decreased $\mathrm{T}$-cell proliferation and cytokine production, limiting the activity of effector cells (40). Additionally, PD-1 has been found to be overstimulated and tumor-specific on T-cells (41). Increased expression of PD-L1 has been shown to result in lower numbers of tumor infiltrating lymphocytes (TILs) (42), and 
higher expression of PD-L1 on tumor cells has been shown to correlate with better response to PD-1 antibody therapy (43).

The second most common checkpoint inhibitor is targeted to a receptor on T-cells known as CTLA-4, which has a high affinity for Tregs. Through its binding interaction with CD80 and CD86 (which limits binding of these to CD28) on antigen presenting cells (APCs), it both promotes Treg immunosuppressive function and sends inhibitory signaling to T-cells (29). With the greater affinity of binding of CTLA-4 to CD80 and CD86 over $\mathrm{CD} 28$, there is a negative feedback loop that leads to T-cell activation and increased CTLA-4 expression in order to prevent autoimmune reactions (44). Finally, murine models have demonstrated that the primary mode of action of antiCTLA-4 drugs is primarily by depleting Tregs via antibodydependent cell-mediated cytotoxicity (45).

Based on the promising preclinical data combining RT and immune checkpoint blockers for non-thoracic malignancies, a number of investigators have explored the efficacy of the combination in preclinical NSCLC models. An initial study of KRAS-mutant NSCLC showed improved survival and tumor shrinkage when combining RT and an anti-PD-1 antibody compared to either modality alone (46). Studies have indicated that this synergy may be the result of RT overcoming PD-1inhibitor resistance via induction of type I interferon (IFN) production leading to enhanced MHC class 1 expression (47). This combination may be promising for translation into the clinic for patients who initially become resistant to anti-PD-1 therapy as a way to increase responsiveness to therapy.

CTLA-4 blockade has also been studied in preclinical lung cancer mouse models in combination with RT (48). Yoshimoto et al. found that RT alone doubled median survival, and the addition of anti-CTLA-4 antibody increased the anti-tumor activity of RT by prolonging the tumor growth delay from 13.1 to 19.5 days. Another study combining RT with anti-CD25 therapy led to decreased Tregs in the spleen and the tumor when compared to monotherapy in an LL/C mouse model (49).

Preclinical studies have also demonstrated potential synergetic effects when combining RT with ICIs. PD-1 blockade enhances T-cells, driving immune response and increasing effector cell activity, while CTLA-4 blockade depletes inhibitory Tregs (50). Additionally, RT alone can influence the immune system in a multitude of ways, some of which enhance tumor response while others render further RT less effective. However, the combination of RT and immune checkpoint blockade has the ability to enhance effects of either one of these therapies alone (and even overcome ineffectiveness of monotherapy), providing an exciting interdisciplinary approach to the management of NSCLC (51).

\section{Preclinical rationale for combining radiation with immunotherapy beyond checkpoint inhibitors}

Modulation of ICIs targeting receptors such as CTLA-4 and PD-L1 is backed by compelling data as demonstrated in recent Checkmate trials $(52,53)$. While promising, checkpoint inhibition as a treatment strategy benefits only a limited population of patients. Accordingly, other immunomodulatory techniques have been investigated including the use of T-cells that have been genetically modified to target cancer specific CARs.

\section{Basic principles of CAR T-cell therapy}

The ability to evade growth suppression and circumvent immunosurveillance is a distinct feature of cancer cells (54). This hallmark characteristic is mediated, in part, by the downregulation of the class $1 \mathrm{MHC}$ proteins. The crux of CAR T-cell therapy is based on genetically engineering a $\mathrm{T}$-cell with a CAR that can recognize cancer cells independent of MHC proteins. While the theory of T-cell mediated therapy is not new, advances in molecular synthesis have elevated this strategy as an exciting therapeutic option (55-58).

Constructing these modified T-cells requires attention to two major components: an intracellular transmembrane component and an extracellular, tumor-recognition component. The development of these genetically engineered T-cells has been characterized by increasingly complex co-stimulatory activation domains within the intracellular component to achieve a sustainable T-cell response. Specifically, the first generation of CAR T-cells contained a $\mathrm{CD}-3 \zeta$ containing domain, while additional domains including CD28, OX40 or 4-1BB now characterize the latest generation of CAR T-cells. Equally, the extracellular component must be designed for a specific target on cancer cells to maximize efficacy while minimizing the potential for "on target, off tumor" effects where nonpathologic tissues are targeted (59).

\section{Challenges to effective CAR T-cell therapy}

The use of CAR T-cells in solid tumors presents the unique 
challenge of effectively trafficking the genetically engineered T-cells to the target and propagating this response in a hostile tumor microenvironment. The mechanisms that govern the trafficking of native T-cells (adhesion, tethering, chemotaxis, and extravasation) are hindered at each phase by factors specific to the tumor microenvironment. For example, tumor-promoted angiogenesis yields vessels with endothelial cells lacking the necessary molecules for T-cells adherence (60). Furthermore, the physical barrier created by the rich stroma of solid tumors hampers the penetration and aggregation of engineered T-cells that lack the enzyme heparanase (61). Moreover, once T-cells have penetrated, molecular signaling in the tumor microenvironment is immunosuppressive and down regulates the recruitment and aggregation of additional T-cells (62-65).

\section{Preclinical data supporting the use of $R T$ with immunotherapy}

Beyond the benefits of direct cytotoxicity, radiotherapy is associated with immunomodulatory effects that can be leveraged in combination with immunotherapy (66). Consequently, this also presents a greater opportunity for targeting specific tumor-associated antigens $(23,64,67)$. Specific examples of radiotherapy-inducing tumorassociated antigens were demonstrated in carcinoembryonic antigen (CEA)- and mesothelin-expressing tumors. Radiotherapy has been shown to upregulate the expression of the Fas gene in CEA-expressing tumor cells. Using a mouse model, Chakraborty et al. highlighted that radiotherapy enhanced Fas gene expression leading to improved antitumor activity in the setting of CEA-based vaccine therapy where T-cell killing proceeds through the Fas/FasL pathway (68). Mesothelin is a cell surface protein overexpressed in mesotheliomas, pancreatic, and ovarian tumors (69). Hassan et al. demonstrated the dose-dependent response of irradiated A4310K5 carcinoma cells resulting in elevated extracellular mesothelin expression and enhanced antitumor activity of an anti-mesothelin immunotoxin against mesothelin-expressing tumor xenografts. Additional examples of increased tumor associated antigens following radiotherapy include c-met and HER2 (70,71). Beyond enhancing tumor-associated antigens, radiotherapy also has been shown to address some of the barriers to effective T-cell therapy related to trafficking and chemotaxis (72-74).

Preclinical studies demonstrating the treatment synergy between CAR T-cell therapy and radiotherapy are encouraging $(75,76)$. Radiotherapy appears to be a complimentary immunomodulator with the potential to help overcome many of the difficulties surrounding effective CAR T-cell therapy and function beyond its cytotoxic effects. While clinical outcomes of this approach are more limited than combining radiotherapy with ICIs, to further optimize the radiotherapy-CAR $\mathrm{T}$ cell combination approach, topics such as the timing of radiation relative to CAR T-cell therapy and the ideal dose and fractionation of radiotherapy will need to be addressed. CART T-cell therapy is currently under investigation for metastatic NSCLC in a phase II clinical trial at the National Institutes of Health (NCT02133196). The combination CAR T-cell therapy and radiation is likely to be explored in future clinical trials that promise to enhance our understanding of this treatment paradigm.

\section{Radiation plus immunotherapy for metastatic NSCLC}

More than half of patients with NSCLC present with distant metastases at the time of diagnosis (77). Unfortunately, long-term survival for patients with stage IV NSCLC is rare with 5-year overall survival (OS) less than 5\% (77). For patients with stage IV NSCLC, RT has historically been used only for palliative purposes and has not been thought to improve survival over chemotherapy alone. However, two recent randomized clinical trials have demonstrated that in appropriately selected NSCLC patients with a low burden of metastatic disease to a limited number of distant sites (oligometastatic), RT can improve progression-free survival (PFS) and potentially OS $(78,79)$. This revelation, along with the preclinical findings of the potential synergy of RT with immunotherapies has opened up an exciting new indication for RT in patients with metastatic NSCLC.

Platinum-based doublet chemotherapy has historically been the standard first-line systemic therapy regimen for patients with metastatic NSCLC without activating mutations in epidermal growth factor receptor (EGFR) or translocations in anaplastic lymphoma kinase (ALK) or ROS1 (80). Taxane-based regimens have historically been utilized as second-line regimens for patients that have progressive disease after receiving a platinum-based regimen (81). In 2015, results of the CheckMate 017 trial revolutionized systemic therapy for NSCLC. That trial randomized patients with metastatic NSCLC who had progressed during or after first-line chemotherapy to docetaxel or the PD-1 inhibitor, nivolumab, found a significant improvement in median OS along with 
significantly fewer serious treatment-related adverse events (AEs) in the nivolumab arm (52). Similarly, the nearly identical KEYNOTE-010 and POPLAR trials found significant improvements in OS and fewer serious AEs in patients receiving the PD-1 inhibitor pembrolizumab, or the PD-L1 inhibitor atezolizumab, respectively, in patients with metastatic NSCLC that had progressed during or after first-line chemotherapy $(82,83)$. PD-L1 expression $\geq 1 \%$ on tumor cells was required in the KEYNOTE-010 trial. Based on the results of these ground-breaking clinical trials, the FDA approved nivolumab, pembrolizumab, and atezolizumab for second-line treatment of metastatic NSCLC.

Two recent clinical trials have demonstrated that ICIs are also effective as first-line therapy for select patients with metastatic NSCLC. The KEYNOTE-024 trial randomized patients with treatment-naïve metastatic NSCLC without sensitizing EGFR mutations or ALK rearrangements and with PD-L1 expression $\geq 50 \%$ to pembrolizumab or platinum-based chemotherapy. The investigators found that response rate and OS were significantly better in the pembrolizumab group compared to the chemotherapy group and fewer severe AEs occurred in the pembrolizumab group. Based on the results of this trial, the FDA approved pembrolizumab for first-line treatment of patients with metastatic NSCLC whose tumors express PD-L1 on $\geq 50 \%$ of tumor cells (84). The KEYNOTE-189 trial randomized patients with treatment-naïve metastatic nonsquamous NSCLC without sensitizing EGFR mutations or ALK rearrangements regardless of $\mathrm{PD}-\mathrm{L} 1$ expression to a platinum-based chemotherapy doublet with pembrolizumab or the same chemotherapy regimen plus placebo. Patients on the pembrolizumab arm had significantly higher OS and PFS regardless of PD-L1 expression levels, with similar rates of severe AEs (85).

The rapid adoption of ICIs for metastatic NSCLC and the frequent need for palliative RT for this patient population have resulted in a number of retrospective analyses reporting the safety and efficacy of combining ICIs with RT. Hubbeling et al. reported no significant difference in RT-related AEs in patients with metastatic NSCLC receiving cranial $\mathrm{RT}$ that previously or concurrently received $\mathrm{PD}-1 / \mathrm{PD}-\mathrm{L} 1$ inhibitors compared to patients receiving cranial RT who were $\mathrm{PD}-1 / \mathrm{PD}-\mathrm{L} 1$ inhibitor naïve (86). This and other early clinical data indicating that combining RT with ICIs may be safe, and the promising preclinical data demonstrating potential increased efficacy with the combination has led to at least 4 ongoing prospective clinical trials evaluating the safety and efficacy of the combination of RT and PD-1/PD-L1 inhibitors for metastatic NSCLC, and an additional 8 trials combining RT with other immunotherapy agents or combinations of ICIs (Table 1). Many studies are evaluating hypofractionated regimens, usually via stereotactic body radiation therapy (SBRT), rather than lower doses of RT typically used in the palliative setting, in hopes of eliciting greater antigen release in an attempt to improve the efficacy of the ICIs. The results of these clinical trials, expected in the next few years, will greatly enhance our understanding of the potential for RT to synergize with ICIs to provide clinically meaningful improvements in outcomes of patients with metastatic NSCLC.

\section{Radiation plus immunotherapy locally advanced and early stage NSCLC}

\section{Locally advanced}

Approximately one-quarter of patients with NSCLC present with locally advanced disease with regional lymph node involvement. For years, these patients have been primarily treated with concurrent platinum-based doublet chemotherapy and daily RT over 6 to 7 weeks. However, long-term disease control rates with this approach are limited. Two years after chemoradiation, nearly half of patients develop distant metastases, and only $30 \%$ will be alive without progressive disease (87). There were not any major advances in this approach for many years with randomized clinical trials of both induction (88) and maintenance chemotherapy (89) failing to consistently demonstrate improvements in OS. Dose escalation with conventional radiation even seems to decrease OS for patients with locally advanced disease (87).

While there have been other studies looking at vaccines as immunotherapy for patients with locally advanced NSCLC $(10,90,91)$, START (Stimulating Targeted Antigenic Response To non-small-cell lung cancer) was the earliest randomized phase III clinical trial to add immunotherapy consolidation after chemoradiation for locally advanced NSCLC (9). It assessed the efficacy of tecemotide, a mucin 1 (MUC1) antigen-specific liposome vaccine injected subcutaneously. In earlier studies, it was capable of inducing a $\mathrm{T}$-cell response in both mouse models and patients. A single-arm phase 2 trial after chemoradiation showed promising results (92). START enrolled 1,513 patients and randomized them in a 2:1 ratio 

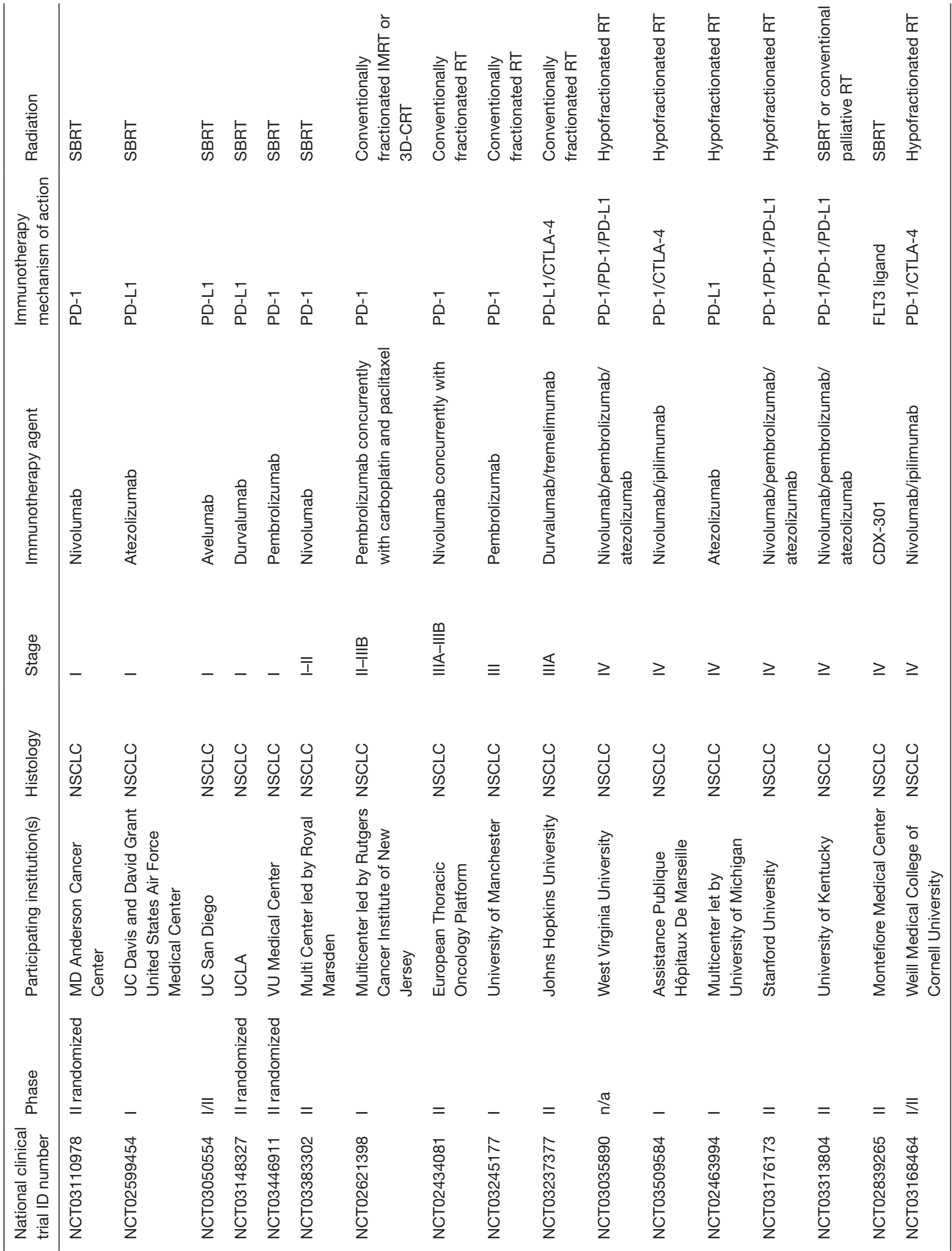


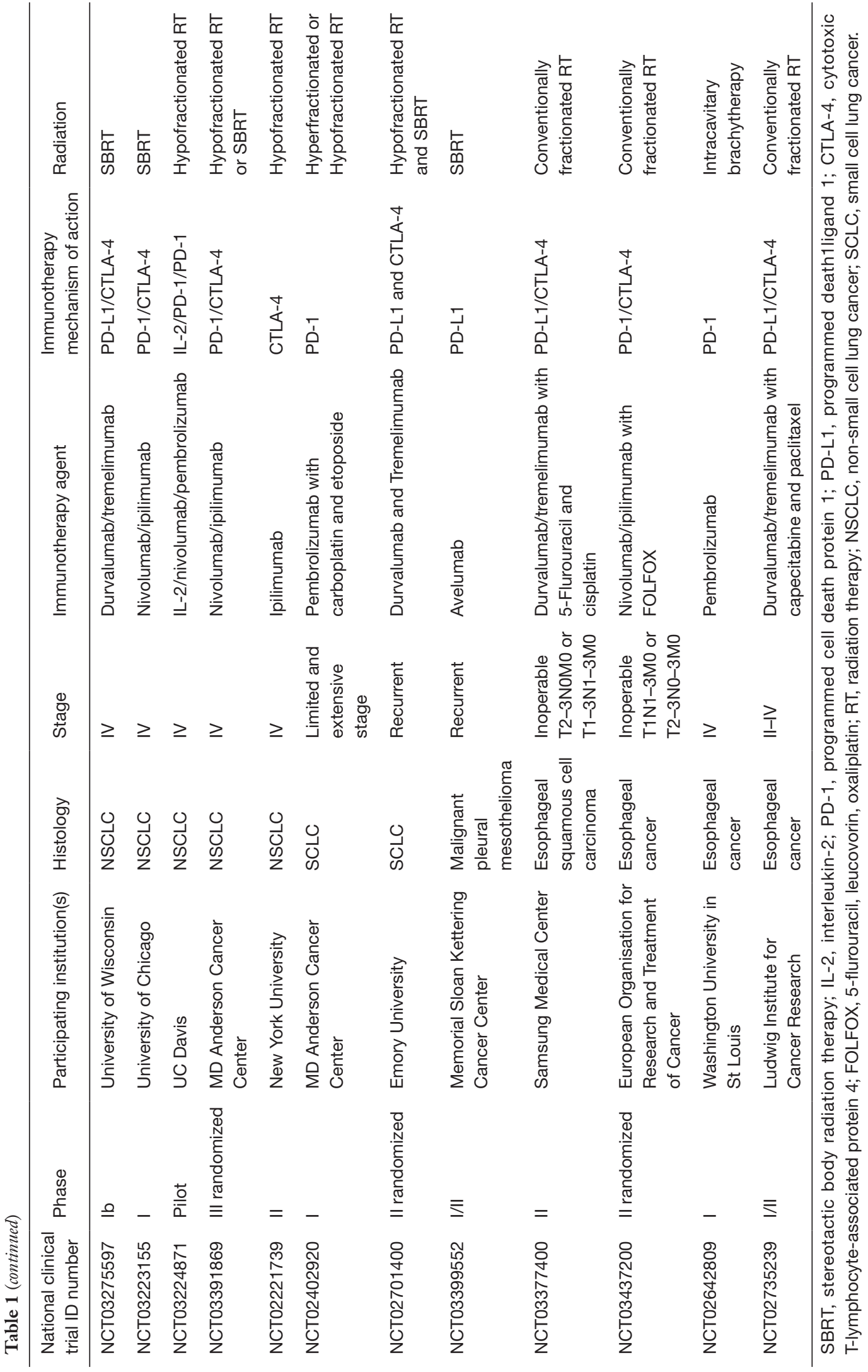


to immunotherapy consolidation versus placebo. This trial showed no significant difference in OS for all patients in the modified intention-to-treat population. However, in a subset analysis of patients who received concurrent chemoradiation therapy, median OS was 30.8 months with immunotherapy compared to 20.6 months with placebo $(\mathrm{P}=0.016)$. In those who received sequential chemotherapy and RT, however, there was no difference in OS $(\mathrm{P}=0.38)$. Importantly, this drug showed no increase in serious grade 3 or 4 AEs (9).

In a similar study design to START, PACIFIC randomized patients to receive consolidation with the antiPD-L1 antibody durvalumab (at a dose of $10 \mathrm{mg}$ given intravenously per kilogram of body weight) or placebo every 2 weeks until disease progression or 12 months, whichever occurred first (93). Durvalumab was given 1-42 days after the conclusion of chemotherapy and RT. In a report from a planned interim analysis, the median PFS from randomization was 16.8 months with immunotherapy versus 5.6 months with placebo (HR 0.52, $\mathrm{P}<0.001$ ). The other primary end-point, OS, has yet to be reported. Secondary end points of response rate, duration of response, and the median time to death or distant metastases were all better with durvalumab. Grade 3 or 4 AEs occurred in $29.9 \%$ of patients receiving durvalumab and $26.1 \%$ of those receiving placebo. The most common grade 3 or $4 \mathrm{AE}$ was pneumonia at $4.4 \%$ with durvalumab and $3.8 \%$ with placebo.

Adoption of consolidation with durvalumab has been mixed globally to date with many national health systems awaiting the OS results before considering it standard of care. The above PFS results, however, led to FDA approval of durvalumab in the United States, as well as incorporation as a standard of care in the National Comprehensive Cancer Network guidelines for patients with stage III disease, performance status $0-1$, and no disease progression after 2 or more cycles of definitive chemoradiation (94). European Society for Medical Oncology guidelines have not yet incorporated durvalumab into national guidelines. A health technology appraisal on this use of durvalumab is expected to be published in January 2019 by the National Institute for Health and Care Excellence in the United Kingdom.

There are other actively recruiting singe-arm trials that are exploring other immunotherapy agents (Table 1). MD Anderson Cancer Center is recruiting to DETERRED, a study exploring the safety of MDPL3280A, a PD-L1 inhibitor, as part of consolidation therapy (NCT02525757). There are two studies ongoing that are moving PD-1 inhibitors earlier in the treatment sequence at the same time as concurrent chemotherapy and RT. In a Rutgers Cancer Institute of New Jersey led multi-center trial, the safety of adding pembrolizumab to carboplatin and paclitaxel for patients with stage II-IIIB NSCLC (NCT02621398) is being tested. A similar phase II study by the European Thoracic Oncology Platform is evaluating pneumonitis rates with the addition of 4 doses of nivolumab given concurrently with standard concurrent chemotherapy (NCT02434081). Both radiation and immunotherapy can cause a similar presentation of pneumonitis, and to date, there are little prospective data on the potential synergistic toxicity of the combination. This combination is, therefore, being approached with caution.

\section{Early stage}

Patients with NSCLC without regional nodal spread can either undergo a resection or be treated with RT. SBRT has emerged over the past decade as an effective treatment with improved disease control and patient quality of life when compared to conventionally fractionated radiation (95). While primary tumor control is high with SBRT, systemic recurrence remains problematic, and the predominant method of failure. However, SBRT has been shown anecdotally to cause an abscopal effect in patients receiving immunotherapy for a variety of solid tumors, where after the administration of radiation to one location, there is a response in tumor(s) at distant sites. There is hope that this combination in early stage NSCLC can reduce the rate of nodal and distant recurrences. Trials are exploring whether the combination of SBRT with immunotherapy can be performed safely, as patients with early stage NSCLC have longer life expectancies and thus are at risk of developing long-term toxicities.

In the United States, there are at least four clinical trials assessing the efficacy and safety of ICIs after SBRT for early-stage NSCLC (Table 1). A multicenter phase I study of atezolizumab, an anti-PD-L1 monoclonal antibody, in combination with SBRT in high-risk early-stage NSCLC is recruiting (NCT02599454) in order to determine the maximum tolerated dose (MTD) of atezolizumab with SBRT. A similar phase I/II study is recruiting at the University of California, San Diego using the PD-L1 monoclonal antibody, avelumab (NCT03050554), and two separate phase II are randomizing patients with earlystage NSCLC to SBRT with or without durvalumab (NCT03148327) or nivolumab (NCT03110978), 
respectively.

In Europe, two trials will explore the tolerability of combining SBRT and PD-1 antibodies (Table 1). Tolerability is largely defined by the rate of grade 3 or greater pneumonitis. At VU University Medical Center in the Netherlands, patients with early stage node-negative NSCLC will be randomized to receive either SBRT alone or SBRT with 2 cycles of pembrolizumab followed by lobectomy (NCT03446911). If the rate of grade 3 or greater pneumonitis is $\leq 10 \%$, the combination will be regarded as safe. In Great Britain, STILE (NCT03383302) is a single arm, multi-center phase II open-label study of nivolumab given within 24 hours of the final fraction of SBRT then every 2 weeks. If the rate of grade 3 or greater pneumonitis exceeds $20 \%$ in the first 6 months, the combination will be deemed unacceptable.

As these small studies attest, combining immunotherapy and RT for early-stage NSCLC is still in its infancy. Challenges with early-stage patients include appropriate selection of the patients that are most likely to benefit from combined modality treatment as well as optimal sequencing and duration of immunotherapy. The optimal radiation dose and fractionation for SBRT alone remain to be determined for peripheral and central tumors, much less when SBRT is combined with immunotherapy. Pseudoprogression after immunotherapy also will likely make assessing response only more challenging based on current RECIST size-based criteria. Nonetheless, there is promising potential synergy between radiation and immunotherapy to reduce systemic failures and improve cure rates in early stage patients (96).

\section{Radiation plus immunotherapy for SCLC}

Despite a plethora of clinical trials for patients with SCLC over the last two decades, little progress has been made and patient outcomes remain poor, with OS ranging between 10 and 30 months depending on extent of disease. Although very responsive to first-line chemotherapy, SCLC frequently relapses, and response to second-line agents is extremely poor (97). Immunotherapy has thus been an exciting development for SCLC as it has the potential to overcome the limitations of chemotherapy by targeting SCLC in a novel way.

The advent of immunotherapy has spurred a search for biomarkers that can predict which patients may best respond to ICIs. PD-L1 expression, though helpful, has modest sensitivity and specificity (84). Numerous preclinical and clinical studies have demonstrated that tumors with a high mutagenic burden, and thus high expression of neoantigens, have high response rates to ICIs, irrespective of levels of PD-L1 tumor expression (11). SCLC has long been known to have a high tumor mutation burden (98). This has been found to be correlated with response to checkpoint inhibitors due to re-awakening of pre-existing strong anti-tumor CD8+ cytotoxic T-cell responses $(98,99)$. There is also some pre-clinical and clinical evidence for an immunomodulatory effect of SCLC tumor cells on host immune system (100-102). The higher propensity of paraneoplastic syndromes in SCLC is also hypothesized to be evidence to suggest that several cross-reacting antibodies recruited by the host immune response target both tumor as well as normal cells. Apart from the immuno-stimulatory effects, there is evidence to suggest that SCLC tumor cells also exhibit a regulatory effect through induction of CD4+ Treg cells and decreased expression of HLA-class 1 antigen on host immunity. This balance between the effector and regulatory effects may distinguish extensive stage SCLC (ES-SCLC) from limited stage SCLC (LS-SCLC) (103).

Both single and combination immunotherapy regimens have been investigated in SCLC. The first agent evaluated in ES-SCLC was the CTLA-4 antibody ipilimumab. A number of studies, including a randomized phase III trial evaluating it in combination with chemotherapeutic agents in the first line setting, showed objective responses but failed to meet their primary survival endpoints (104). The KEYNOTE 028 study evaluated pembrolizumab in previously treated ES-SCLC patients. It showed an impressive response rate (RR) of $29 \%$ in unselected and $33.3 \%$ in PD-L1 expression-positive patients. Responses were rapid and durable with a median duration of response of 19.4 months and OS of 9.7 months. It was tolerated well with an acceptable toxicity profile (105). The CheckMate 032 trial, which compared the combination of nivolumab and ipilimumab with single-agent nivolumab as secondline therapy for ES-SCLC patients, showed a RR of $25 \%$, compared to $11 \%$ with nivolumab alone and a near doubling of OS (7.9 vs. 4.1 months, respectively) at the expense of increased toxicity (106).

Although encouraging, priming with chemotherapy alone may generate only subpar neoantigen expression and a subsequent inadequate response. In this context, radiation may enhance tumor immunogenicity by modulating the immune response both at the systemic level and at the level of the tumor microenvironment. There are currently three trials evaluating the combination of radiation with ICIs (Table 1). Investigators at MD Anderson Cancer 
Center are performing a phase-I dose escalation study of pembrolizumab in combination with carboplatin, etoposide, and RT in LS-SCLC and ES-SCLC (NCT02402920). In Europe, the ongoing phase II randomized multicenter STIMULI study is comparing standard chemoradiation and prophylactic cranial irradiation followed by either observation alone or 4 cycles of ipilimumab and nivolumab followed by maintenance nivolumab for patients with LSSCLC (NCT02046733). Finally, Emory University is conducting a randomized phase II study (NCT02701400) evaluating the combination of tremelimumab and durvalumab with or without hypofractionated RT or SBRT in patients with relapsed SCLC. These studies will address the toxicity concerns of combining these agents with radiation, especially in the context of the relatively large target volumes seen in SCLC as well as providing valuable data on the appropriate dose, combination, and timing of the treatments.

\section{Radiation plus immunotherapy for mesothelioma}

MPM is a rare disease with poor OS and limited effective treatment options. The development of metastatic disease is common, and there have not been significant strides in cytotoxic chemotherapies in recent years. Patients with MPM often have a large burden of disease and poor performance status, thus the discovery of effective immunotherapies has long been of interest. IL-2, IFN-alfa $2 \mathrm{a}$ and IFN-alfa $2 \mathrm{~b}$ have been evaluated with mixed results (107-109). A recent trial of chemotherapy and adenovirus containing IFN-alfa $2 \mathrm{~b}$ in previously treated patients has resulted in an encouraging 21.5 months OS (109). Unfortunately, the CTLA-4 inhibitor tremelimumab failed to show an OS benefit in a recent phase III trial $(110,111)$. Anti PD-1/PD-L1 drugs, however, have shown promising results in a series of small studies. Phase-I/II studies incorporating pembrolizumab, nivolumab, and avelumab have shown $9.4-20 \%$ partial response rates with stabilization of disease in $50 \%$ of patients (112-114). Combined PD$\mathrm{L} 1$ inhibition is also being explored in two ongoing clinical trials (NCT03048474 and NCT02899299). A report from the University of Toronto found that the growth of tumors in a murine mesothelioma model was significantly reduced by hypofractionated radiation and combining radiation with a CTLA-4 inhibitor enhanced the effect in the irradiated and unirradiated tumors (115). Combining radiotherapy and immunotherapy for mesothelioma is a promising treatment strategy (116), and clinical data for the combination of radiation and ICIs are forthcoming. A single arm phase II study has recently been initiated by Memorial Sloan Kettering Cancer Center and will evaluate response rates with Avelumab and SBRT in MPM (NCT03399552) (Table 1).

\section{Radiation plus immunotherapy for esophageal cancer}

Trimodality therapy consisting of concurrent chemoradiation followed by surgical resection is the standard of care for locally advanced esophageal cancer patients who are surgical candidates. The 5-year OS with this approach ranges from $39-47 \%(117,118)$. In patients that are medically inoperable or have unresectable disease, definitive chemoradiation is recommended; however, survival rates are poor and persistence of locoregional disease occurs in nearly half of patients $(119,120)$. More aggressive treatments such as radiation dose-escalation and the addition of targeted systemic agents against receptors commonly expressed in esophageal cancer such as EGFR have so far failed to improve patient outcomes $(120,121)$.

$\mathrm{PD}-\mathrm{L} 1$ expression is present in $45 \%$ of esophageal cancer tissues and is associated with more locally aggressive disease and decreased survival (122). Irradiated tumors have been associated with an increased expression of PDL1 leading to suppression of anti-tumoral activity of T-cells (16). Consequently, monoclonal antibodies directed against PD-L1 receptors may act synergistically with RT in killing tumor cells. This is the subject of several ongoing phase I-II trials evaluating safety and efficacy of the combination of chemoradiation with ICIs in both the metastatic/inoperable (NCT03377400, NCT03437200, NCT02642809) and neoadjuvant settings (NCT02735239) (Table 1).

\section{Radiation plus immunotherapy for thymoma}

Thymomas represent $20 \%$ of all primary mediastinal tumors (123). Surgical resection is the primary treatment. Postoperative radiation is recommended in the presence of positive margins and for advanced stages (4). While survival outcomes are generally good, intrathoracic failures after definitive treatment for thymoma can occur in approximately up to one-quarter of patients (124). Due to its rarity, there is a dearth of understanding of the molecular biology of these tumors, and immunotherapy approaches are limited to small patient cohorts. There are some studies 
suggesting $\mathrm{PD}-\mathrm{L} 1$ expression has higher prevalence in more aggressive histologies (i.e., B1-3 thymomas and thymic carcinomas) and in higher Masaoka stages $(125,126)$. Avelumab was evaluated in 7 patients with locally advanced thymoma and a partial response was observed in 4 of these patients (127). There are two ongoing phase II trials evaluating the efficacy of ICIs in thymomas (NCT02721732 and NCT02607631); however, none currently are in combination with radiation. The efficacy of immunotherapy combined with radiation will likely be of greater interest as our understanding of thymoma tumor biology and its interaction with the immune system improves.

\section{Conclusions}

The combination of immunotherapy and RT has the potential to revolutionize treatments for thoracic malignancies. Preclinical data have demonstrated impressive synergy between the two therapies that appears to extend beyond the irradiated target. For patients with advanced NSCLC, recent clinical trials incorporating ICIs have exhibited dramatic improvements in outcomes compared to conventional chemotherapies. We anxiously await results from ongoing and future preclinical research and clinical trials to better define the optimal approaches to combining these two pillars of cancer care.

\section{Acknowledgements}

None.

\section{Footnote}

Conflicts of Interest: Dr. Badiyan and Dr. Simone have speaker's honoraria from Varian Medical Systems.

\section{References}

1. Siegel RL, Miller KD, Jemal A. Cancer statistics, 2018. CA Cancer J Clin 2018;68:7-30.

2. Vogelzang NJ, Rusthoven JJ, Symanowski J, et al. Phase III study of pemetrexed in combination with cisplatin versus cisplatin alone in patients with malignant pleural mesothelioma. J Clin Oncol 2003;21:2636-44.

3. Suntharalingam $M$, Winter K, Ilson D, et al. Effect of the Addition of Cetuximab to Paclitaxel, Cisplatin, and Radiation Therapy for Patients With Esophageal Cancer. JAMA Oncol 2017;3:1520-8.
4. Jackson MW, Palma DA, Camidge DR, et al. The Impact of Postoperative Radiotherapy for Thymoma and Thymic Carcinoma. J Thorac Oncol 2017;12:734-44.

5. Couzin-Frankel J. Breakthrough of the year 2013. Cancer immunotherapy. Science 2013;342:1432-3.

6. Smyth MJ. Multiple approaches to immunotherapy The new pillar of cancer treatment. Immunol. Cell Biol 2017;95:323-4.

7. Weber JS, D'Angelo SP, Minor D, et al. Nivolumab versus chemotherapy in patients with advanced melanoma who progressed after anti-CTLA-4 treatment (CheckMate 037): A randomised, controlled, open-label, phase 3 trial. Lancet Oncol 2015;16:375-84.

8. Hodi FS, Chesney J, Pavlick AC, et al. Combined nivolumab and ipilimumab versus ipilimumab alone in patients with advanced melanoma: 2-year overall survival outcomes in a multicentre, randomised, controlled, phase 2 trial. Lancet Oncol 2016;17:1558-68.

9. Butts C, Socinski MA, Mitchell PL, et al. Tecemotide (L-BLP25) versus placebo after chemoradiotherapy for stage III non-small-cell lung cancer (START): A randomised, double-blind, phase 3 trial. Lancet Oncol 2014;15:59-68.

10. Brunsvig PF, Kyte JA, Kersten C, et al. Telomerase peptide vaccination in NSCLC: A phase II trial in stage III patients vaccinated after chemoradiotherapy and an 8-year update on a phase I/II trial. Clin Cancer Res 2011;17:6847-57.

11. Hellmann MD, Ciuleanu TE, Pluzanski A, et al. Nivolumab plus Ipilimumab in Lung Cancer with a High Tumor Mutational Burden. N Engl J Med 2018;378:2093-104.

12. Wolchok JD, Chiarion-Sileni V, Gonzalez R, et al. Overall Survival with Combined Nivolumab and Ipilimumab in Advanced Melanoma. N Engl J Med 2017;377:1345-56.

13. Twyman-Saint Victor C, Rech AJ, Maity A, et al. Radiation and Dual Checkpoint Blockade Activates Non-Redundant Immune Mechanisms in Cancer. Nature 2015;520:373-7.

14. Theurich S, Rothschild SI, Hoffmann M, et al. Local Tumor Treatment in Combination with Systemic Ipilimumab Immunotherapy Prolongs Overall Survival in Patients with Advanced Malignant Melanoma. Cancer Immunol Res 2016;4:744-54.

15. Demaria S, Coleman CN, Formenti SC. Radiotherapy: Changing the Game in Immunotherapy. Trends Cancer 2016;2:286-94.

16. Deng L, Liang H, Burnette B, et al. Irradiation and antiPD-L1 treatment synergistically promote antitumor immunity in mice. J Clin Invest 2014;124:687-95. 
17. Golden EB, Demaria S, Schiff PB, et al. An abscopal response to radiation and ipilimumab in a patient with metastatic non-small cell lung cancer. Cancer Immunol Res 2013;1:365-72.

18. Correction to Lancet Oncol 2017; 18: 895-903. Lancet Oncol 2017; 18:e371.

19. Suzuki T, Chow CW, Downey GP. Role of innate immune cells and their products in lung immunopathology. Int $\mathrm{J}$ Biochem Cell Biol 2008;40:1348-61.

20. Diamond G, Legarda D, Ryan LK. The innate immune response of the respiratory epithelium. Immunol Rev 2000;173:27-38.

21. Gentles AJ, Newman AM, Liu CL, et al. The prognostic landscape of genes and infiltrating immune cells across human cancers. Nat Med 2015;21:938-45.

22. Schoenhals JE, Seyedin SN, Anderson C, et al. Uncovering the immune tumor microenvironment in non-small cell lung cancer to understand response rates to checkpoint blockade and radiation. Transl Lung Cancer Res 2017;6:148-58.

23. Reits EA, Hodge JW, Herberts CA, et al. Radiation modulates the peptide repertoire, enhances $\mathrm{MHC}$ class I expression, and induces successful antitumor immunotherapy. J Exp Med 2006;203:1259-71.

24. Dancea HC, Shareef MM, Ahmed MM. Role of Radiationinduced TGF-beta Signaling in Cancer Therapy. Mol Cell Pharmacol 2009;1:44-56.

25. Vatner RE, Formenti SC. Myeloid-Derived Cells in Tumors: Effects of Radiation. Semin Radiat Oncol 2015;25:18-27.

26. Ahn GO, Tseng D, Liao CH, et al. Inhibition of Mac1 (CD11b/CD18) enhances tumor response to radiation by reducing myeloid cell recruitment. Proc Natl Acad Sci 2010;107:8363-8.

27. Filatenkov A, Baker J, Mueller AM, et al. Ablative tumor radiation can change the tumor immune cell microenvironment to induce durable complete remissions. Clin Cancer Res 2015;21:3727-39.

28. Simone CB, Burri SH, Heinzerling JH. Novel radiotherapy approaches for lung cancer: combining radiation therapy with targeted and immunotherapies. Transl Lung Cancer Res 2015;4:545-52.

29. Pardoll DM. The blockade of immune checkpoints in cancer immunotherapy. Nat Rev Cancer 2012;12:252-64.

30. Ishida Y, Agata Y, Shibahara K, et al. Induced expression of PD-1, a novel member of the immunoglobulin gene superfamily, upon programmed cell death. EMBO J 1992;11:3887-95.
31. Freeman GJ, Long AJ, Iwai Y, et al. Engagement of the Pd-1 Immunoinhibitory Receptor by a Novel B7 Family Member Leads to Negative Regulation of Lymphocyte Activation. J Exp Med 2000;192:1027-34.

32. Keir ME, Liang SC, Guleria I, et al. Tissue expression of PD-L1 mediates peripheral T cell tolerance. J Exp Med 2006;203:883-95.

33. Nishimura H, Okazaki T, Tanaka Y, et al. Autoimmune dilated cardiomyopathy in $\mathrm{PD}-1$ receptor-deficient mice. Science 2001;291:319-22.

34. Nishimura H, Nose M, Hiai H, et al. Development of lupus-like autoimmune diseases by disruption of the PD-1 gene encoding an ITIM motif-carrying immunoreceptor. Immunity 1999;11:141-51.

35. Okazaki T, Honjo T. PD-1 and PD-1 ligands: From discovery to clinical application. Int Immunol 2007;19:813-24.

36. Keir ME, Butte MJ, Freeman GJ, et al. PD-1 and Its Ligands in Tolerance and Immunity. Annu Rev Immunol 2008;26:677-704.

37. Dong H, Strome SE, Salomao DR, et al. Tumor-associated B7-H1 promotes T-cell apoptosis: A potential mechanism of immune evasion. Nat Med 2002;8:793-800.

38. Blank C, Brown I, Peterson AC, et al. PD-L1/B7H-1 Inhibits the Effector Phase of Tumor Rejection by T Cell Receptor (TCR) Transgenic CD8+T Cells. Cancer Res 2004;64:1140-5.

39. Taube JM, Anders RA, Young GD, et al. Colocalization of inflammatory response with B7-H1 expression in human melanocytic lesions supports an adaptive resistance mechanism of immune escape. Sci Transl Med 2012;4:127ra37.

40. Butte MJ, Keir ME, Phamduy TB, et al. Programmed Death-1 Ligand 1 Interacts Specifically with the B7-1 Costimulatory Molecule to Inhibit T Cell Responses. Immunity 2007;27:111-22.

41. Gros A, Robbins PF, Yao X, et al. PD-1 identifies the patient-specific CD8+tumor-reactive repertoire infiltrating human tumors. J Clin Invest 2014;124:2246-59.

42. Konishi J, Yamazaki K, Azuma M, et al. B7-H1 expression on non-small cell lung cancer cells and its relationship with tumor-infiltrating lymphocytes and their PD-1 expression. Clin. Cancer Res 2004;10:5094-100.

43. Topalian SL, Hodi FS, Brahmer JR, et al. Safety, Activity, and Immune Correlates of Anti-PD-1 Antibody in Cancer. N Engl J Med 2012;366:2443-54.

44. Perkins D, Wang Z, Donovan C, et al. Regulation of CTLA-4 expression during $\mathrm{T}$ cell activation. $\mathrm{J}$ Immunol 
1996;156:4154-9.

45. Selby MJ, Engelhardt JJ, Quigley M, et al. Anti-CTLA-4 Antibodies of IgG2a Isotype Enhance Antitumor Activity through Reduction of Intratumoral Regulatory T Cells. Cancer Immunol Res 2013;1:32-42.

46. Herter-Sprie GS, Koyama S, Korideck H, et al. Synergy of radiotherapy and PD-1 blockade in Kras-mutant lung cancer. JCI Insight 2016;1:e87415.

47. Wang X, Schoenhals JE, Li A, et al. Suppression of Type I IFN Signaling in Tumors Mediates Resistance to AntiPD-1 Treatment That Can Be Overcome by Radiotherapy. Cancer Res 2017;77:839-50.

48. Yoshimoto Y, Suzuki Y, Mimura K, et al. Radiotherapyinduced anti-tumor immunity contributes to the therapeutic efficacy of irradiation and can be augmented by CTLA-4 blockade in a mouse model. PLoS One 2014;9:e92572.

49. Son CH, Bae JH, Shin DY, et al. Combination effect of regulatory $\mathrm{T}$-cell depletion and ionizing radiation in mouse models of lung and colon cancer. Int J Radiat Oncol Biol Phys 2015;92:390-8.

50. Herzberg B, Campo MJ, Gainor JF. Immune Checkpoint Inhibitors in Non-Small Cell Lung Cancer. Oncologist 2017;22:81-8.

51. Sharabi AB, Lim M, DeWeese TL, et al. Radiation and checkpoint blockade immunotherapy: Radiosensitisation and potential mechanisms of synergy. Lancet Oncol 2015;16:e498-e509.

52. Borghaei H, Paz-Ares L, Horn L, et al. Nivolumab versus Docetaxel in Advanced Nonsquamous Non-Small-Cell Lung Cancer. N Engl J Med 2015;373:1627-39.

53. Brahmer J, Reckamp KL, Baas P, et al. Nivolumab versus Docetaxel in Advanced Squamous-Cell Non-Small-Cell Lung Cancer. N Engl J Med 2015;373:123-35.

54. Hanahan D, Weinberg RA. Hallmarks of cancer: The next generation. Cell 2011;144:646-74.

55. Gross G, Waks T, Eshhar Z. Expression of immunoglobulin-T-cell receptor chimeric molecules as functional receptors with antibody-type specificity. Proc. Natl. Acad. Sci 1989;86:10024-8.

56. Kalos M, Levine BL, Porter DL, et al. T cells with chimeric antigen receptors have potent antitumor effects and can establish memory in patients with advanced leukemia. Sci Transl Med 2011;3:95ra73.

57. Carpenito C, Milone MC, Hassan R, et al. Control of large, established tumor xenografts with genetically retargeted human T cells containing CD28 and CD137 domains. Proc Natl Acad Sci 2009;106:3360-5.
58. Milone MC, Fish JD, Carpenito C, et al. Chimeric receptors containing CD137 signal transduction domains mediate enhanced survival of $\mathrm{T}$ cells and increased antileukemic efficacy in vivo. Mol Ther 2009;17:1453-64.

59. Bonifant CL, Jackson HJ, Brentjens RJ, et al. Toxicity and management in CAR T-cell therapy. Mol Ther Oncolytics 2016;3:16011.

60. Griffioen AW, Damen CA, Martinotti S, et al. Endothelial intercellular adhesion molecule-1 expression is suppressed in human malignancies: The role of angiogenic factors. Cancer Res 1996;56:1111-7.

61. Caruana I, Savoldo B, Hoyos V, et al. Heparanase promotes tumor infiltration and antitumor activity of CAR-redirected T lymphocytes. Nat Med 2015;21:524-9.

62. Marvel D, Gabrilovich DI. Myeloid-derived suppressor cells in the tumor microenvironment: expect the unexpected. J Clin Investig 2015;125:3356-64.

63. Gabrilovich DI. Myeloid-Derived Suppressor Cells. Cancer Immunol Res 2017;5:3-8.

64. Pandiyan $\mathrm{P}$, Zheng L, Ishihara $\mathrm{S}$, et al. CD4+CD25+Foxp3+ regulatory T cells induce cytokine deprivation-mediated apoptosis of effector CD4+ T cells. Nat Immunol 2007;8:1353-62.

65. Zou W. Regulatory T cells, tumour immunity and immunotherapy. Nat Rev Immunol 2006;6:295-307.

66. Lugade AA, Moran JP, Gerber SA, et al. Local Radiation Therapy of B16 Melanoma Tumors Increases the Generation of Tumor Antigen-Specific Effector Cells That Traffic to the Tumor. J Immunol 2005;174:7516-23.

67. Chakraborty M, Abrams SI, Coleman CN, et al. External beam radiation of tumors alters phenotype of tumor cells to render them susceptible to vaccine-mediated $\mathrm{T}$-cell killing. Cancer Res 2004;64:4328-37.

68. Chakraborty M, Abrams SI, Camphausen K, et al. Irradiation of Tumor Cells Up-Regulates Fas and Enhances CTL Lytic Activity and CTL Adoptive Immunotherapy. J Immunol 2003;170:6338-47.

69. Hassan R, Williams-Gould J, Steinberg SM, et al. Tumordirected radiation and the immunotoxin SS1P in the treatment of mesothelin-expressing tumor xenografts. Clin. Cancer Res 2006;12:4983-8.

70. Bhardwaj V, Zhan Y, Cortez MA, et al. C-met inhibitor MK-8003 radiosensitizes c-met-expressing non-small-cell lung cancer cells with radiation-induced c-met-expression. J Thorac Oncol 2012;7:1211-7.

71. Cao N, Li S, Wang Z, et al. NF-kappaB-mediated HER2 overexpression in radiation-adaptive resistance. Radiat Res 2009;171:9-21. 
72. Siva S, MacManus M, Kron T, et al. A pattern of early radiation-induced inflammatory cytokine expression is associated with lung toxicity in patients with non-small cell lung cancer. PLoS One 2014;9:e109560.

73. Matsumura S, Wang B, Kawashima N, et al. RadiationInduced CXCL16 Release by Breast Cancer Cells Attracts Effector T Cells. J Immunol 2008;181:3099-107.

74. Ganss R, Ryschich E, Klar E, et al. Combination of T-cell therapy and trigger of inflammation induces remodeling of the vasculature and tumor eradication. Cancer Res 2002;62:1462-70.

75. Weiss T, Weller M, Guckenberger M, et al. NKG2Dbased CAR T cells and radiotherapy exert synergistic efficacy in glioblastoma. Cancer Res 2018;78:1031-43.

76. Flynn JP, O'Hara MH, Gandhi SJ. Preclinical rationale for combining radiation therapy and immunotherapy beyond checkpoint inhibitors (i.e., CART). Transl. Lung Cancer Res 2017;6:159-68.

77. Noone A, Howlader N, Krapcho M, et al. SEER Cancer Statistics Review, 1975-2015, National Cancer Institute. Based November 2017 SEER data submission, posted to SEER web site, April 2018.

78. Gomez DR, Blumenschein GR, Lee JJ, et al. Local consolidative therapy versus maintenance therapy or observation for patients with oligometastatic non-smallcell lung cancer without progression after first-line systemic therapy: a multicentre, randomised, controlled, phase 2 study. Lancet Oncol 2016;17:1672-82.

79. Iyengar P, Tumati V, Gerber D, et al. Consolidative Radiotherapy for Limited Metastatic Non-Small Cell Lung Cancer: A Randomized Phase 2 Trial. Int J Radiat Oncol Biol Phys 2017;99:1314.

80. Noonan KL, Ho C, Laskin J, et al. The Influence of the Evolution of First-Line Chemotherapy on Steadily Improving Survival in Advanced Non-Small-Cell Lung Cancer Clinical Trials. J Thorac Oncol 2015;10:1523-31.

81. Carnio S, Novello S, Mele T, et al. Extending Survival of Stage IV Non-Small Cell Lung Cancer. Semin Oncol 2014;41:69-92.

82. Herbst RS, Baas P, Kim DW, et al. Pembrolizumab versus docetaxel for previously treated, PD-L1-positive, advanced non-small-cell lung cancer (KEYNOTE-010): A randomised controlled trial. Lancet 2016;387:1540-50.

83. Fehrenbacher L, Spira A, Ballinger M, et al. Atezolizumab versus docetaxel for patients with previously treated non-small-cell lung cancer (POPLAR): A multicentre, open-label, phase 2 randomised controlled trial. Lancet 2016;387:1837-46.
84. Reck M, Rodríguez-Abreu D, Robinson AG, et al. Pembrolizumab versus Chemotherapy for PD-L1Positive Non-Small-Cell Lung Cancer. N Engl J Med 2016;375:1823-33.

85. Gandhi L, Delvys R-A, Gadgeel S, et al. Pembrolizumab plus Chemotherapy in Metastatic Non-Small-Cell Lung Cancer. N Engl J Med 2018. [Epub ahead of print].

86. Hubbeling HG, Schapira EF, Horick NK, et al. Safety of Combined PD-1 Pathway Inhibition and Intracranial Radiation Therapy in Non-Small Cell Lung Cancer. J Thorac Oncol 2018;13:550-8.

87. Bradley JD, Paulus R, Komaki R, et al. Standard-dose versus high-dose conformal radiotherapy with concurrent and consolidation carboplatin plus paclitaxel with or without cetuximab for patients with stage IIIA or IIIB nonsmall-cell lung cancer (RTOG 0617): A randomised, twoby-two factorial p. Lancet Oncol 2015;16:187-99.

88. Vokes EE, Herndon JE, Kelley MJ, et al. Induction chemotherapy followed by chemoradiotherapy compared with chemoradiotherapy alone for regionally advanced unresectable stage III non-small-cell lung cancer: Cancer and leukemia group B. J Clin Oncol 2007;25:1698-704.

89. Tsujino K, Kurata T, Yamamoto S, et al. Is consolidation chemotherapy after concurrent chemo-radiotherapy beneficial for patients with locally advanced non-small-cell lung cancer? A pooled analysis of the literature. J Thorac Oncol 2013;8:1181-9.

90. Hansen GL, Gaudernack G, Brunsvig PF, et al. Immunological factors influencing clinical outcome in lung cancer patients after telomerase peptide vaccination. Cancer Immunol. Immunother 2015;64:1609-21.

91. Vansteenkiste JF, Cho BC, Vanakesa T, et al. Efficacy of the MAGE-A3 cancer immunotherapeutic as adjuvant therapy in patients with resected MAGE-A3-positive nonsmall-cell lung cancer (MAGRIT): a randomised, doubleblind, placebo-controlled, phase 3 trial. Lancet Oncol 2016;17:822-35.

92. Butts C, Murray RN, Smith CJ, et al. A multicenter openlabel study to assess the safety of a new formulation of BLP25 liposome vaccine in patients with unresectable stage III non-small-cell lung cancer. Clin Lung Cancer 2010;11:391-5.

93. Antonia SJ, Villegas A, Daniel D, et al. Durvalumab after Chemoradiotherapy in Stage III Non-Small-Cell Lung Cancer. N Engl J Med 2017;377:1919-29.

94. National Comprehensive Cancer Network. Non-Small Cell Lung Cancer. Version 3.2018. Natl Compr Cancer Network 2018. 
95. Nyman J, Hallqvist A, Lund JÅ, et al. SPACE - A randomized study of SBRT vs conventional fractionated radiotherapy in medically inoperable stage I NSCLC. Radiother Oncol 2016;121:1-8.

96. Taunk NK, Rimner A, Culligan M, et al. Immunotherapy and radiation therapy for operable early stage and locally advanced non-small cell lung cancer. Transl Lung Cancer Res 2017;6:178-85.

97. Spigel DR, Socinski MA. Rationale for chemotherapy, immunotherapy, and checkpoint blockade in SCLC: Beyond traditional treatment approaches. J Thorac Oncol 2013;8:587-98.

98. George J, Lim JS, Jang SJ, et al. Comprehensive genomic profiles of small cell lung cancer. Nature 2015;524:47-53.

99. Peifer M, Fernández-Cuesta L, Sos ML, et al. Integrative genome analyses identify key somatic driver mutations of small-cell lung cancer. Nat Genet 2012;44:1104-10.

100. Wang W, Hodkinson P, McLaren F, et al. Small cell lung cancer tumour cells induce regulatory $\mathrm{T}$ lymphocytes, and patient survival correlates negatively with FOXP3 + cells in tumour infiltrate. Int J Cancer 2012;131:E928-37.

101. Wang W, Hodkinson P, McLaren F, et al. Histologic Assessment of Tumor-Associated CD45 + Cell Numbers Is an Independent Predictor of Prognosis in Small Cell Lung Cancer. Chest 2013;143:146-51.

102. Tsuchida T, Yamane H, Ochi N, et al. Cytotoxicity of activated natural killer cells and expression of adhesion molecules in small-cell lung cancer. Anticancer Res 2012;32:887-92.

103. Koyama K, Kagamu H, Miura S, et al. Reciprocal CD4+ T-cell balance of effector CD62Llow CD4+ and CD62LhighCD25+ CD4+ regulatory T cells in small cell lung cancer reflects disease stage. Clin Cancer Res 2008;14:6770-9

104. Reck M, Luft A, Szczesna A, et al. Phase III randomized trial of ipilimumab plus etoposide and platinum versus placebo plus etoposide and platinum in extensive-stage small-cell lung cancer. J Clin Oncol 2016;34:3740-8.

105. Ott PA, Bang YJ, Berton-Rigaud D, et al. Safety and antitumor activity of pembrolizumab in advanced programmed death ligand 1-positive endometrial cancer: Results from the KEYNOTE-028 study. J Clin Oncol 2017;35:2535-41.

106. Antonia SJ, López-Martin JA, Bendell J, et al. Nivolumab alone and nivolumab plus ipilimumab in recurrent smallcell lung cancer (CheckMate 032): a multicentre, openlabel, phase 1/2 trial. Lancet Oncol 2016;17:883-95.

107.Lucchi M, Chella A, Melfi F, et al. Four-modality therapy in malignant pleural mesothelioma: a phase II study. J

Thorac Oncol 2007;2:237-42.

108. Alì G, Boldrini L, Lucchi M, et al. Treatment with interleukin-2 in malignant pleural mesothelioma: Immunological and angiogenetic assessment and prognostic impact. Br J Cancer 2009;101:1869-75.

109. Sterman DH, Alley E, Stevenson JP, et al. Pilot and Feasibility Trial Evaluating Immuno-Gene Therapy of Malignant Mesothelioma Using Intrapleural Delivery of Adenovirus-IFN $\alpha$ Combined with Chemotherapy. Clin Cancer Res 2016;22:3791-800.

110. Calabrò L, Morra A, Fonsatti E, et al. Efficacy and safety of an intensified schedule of tremelimumab for chemotherapy-resistant malignant mesothelioma: An open-label, single-arm, phase 2 study. Lancet Respir Med 2015;3:301-9.

111. Kindler HL, Scherpereel A, Calabrò L, et al. Tremelimumab as second- or third-line treatment of unresectable malignant mesothelioma (MM): Results from the global, double-blind, placebo-controlled DETERMINE study. J Clin Oncol 2016;34:abstr 8502.

112.Alley EW, Lopez J, Santoro A, et al. Clinical safety and activity of pembrolizumab in patients with malignant pleural mesothelioma (KEYNOTE-028): preliminary results from a non-randomised, open-label, phase $1 \mathrm{~b}$ trial. Lancet Oncol 2017;18:623-30.

113. Hassan R, Thomas A, Nemunaitis JJ, et al. Avelumab in patients with previously treated mesothelioma: Updated phase $1 \mathrm{~b}$ results from the JAVELIN Solid Tumor trial. J Clin Oncol 2018;36:abstr 166.

114. Quispel-Janssen J, Zago G, Schouten R, et al. A Phase II Study of Nivolumab in Malignant Pleural Mesothelioma (NivoMes): with Translational Research (TR) Biopsies. J Thorac Oncol 2017;12:s292-3.

115.Wu L, Wu MO, De la Maza L, et al. Targeting the inhibitory receptor CTLA-4 on T cells increased abscopal effects in murine mesothelioma model. Oncotarget 2015;6:12468-80.

116.Alley EW, Katz SI, Cengel KA, et al. Immunotherapy and radiation therapy for malignant pleural mesothelioma. Transl Lung Cancer Res 2017;6:212-9.

117.van Hagen P, Hulshof MCCM, van Lanschot JJB, et al. Preoperative Chemoradiotherapy for Esophageal or Junctional Cancer. N Engl J Med 2012;366:2074-84.

118. Tepper J, Krasna MJ, Niedzwiecki D, et al. Phase III trial of trimodality therapy with cisplatin, fluorouracil, radiotherapy, and surgery compared with surgery alone for esophageal cancer: CALGB 9781. J Clin Oncol 
2008;26:1086-92.

119. Cooper JS, Guo MD, Herskovic A, et al.

Chemoradiotherapy of locally advanced esophageal cancer: long-term follow-up of a prospective randomized trial (RTOG 85-01). Radiation Therapy Oncology Group. JAMA 1999;281:1623-7.

120.Minsky BD, Pajak TF, Ginsberg RJ, et al. INT 0123

(Radiation therapy oncology group 94-05) phase III trial of combined-modality therapy for esophageal cancer: Highdose versus standard-dose radiation therapy. J Clin Oncol 2002;20:1167-74.

121. Suntharalingam M, Winter K, Ilson D, et al. Effect of the addition of cetuximab to paclitaxel, cisplatin, and radiation therapy for patients with esophageal cancer the NRG oncology rtog 0436 phase 3 randomized clinical trial. JAMA Oncol 2017;3:1520-8.

122. Chen MF, Chen PT, Chen WC, et al. The role of PD$\mathrm{L} 1$ in the radiation response and prognosis for esophageal squamous cell carcinoma related to IL-6 and T-cell

Cite this article as: Badiyan SN, Roach MC, Chuong MD, Rice SR, Onyeuku NE, Remick J, Chilukuri S, Glass E, Mohindra P, Simone CB 2nd. Combining immunotherapy with radiation therapy in thoracic oncology. J Thorac Dis 2018;10(Suppl 21):S2492-S2507. doi: 10.21037/jtd.2018.05.73 immunosuppression. Oncotarget 2016;7:7913-24.

123. Duwe BV, Sterman DH, Musani AI. Tumors of the mediastinum. Chest 2005;128:2893-909.

124. Rimner A, Gomez DR, Wu AJ, et al. Failure patterns relative to radiation treatment fields for stage II-IV thymoma. J Thorac Oncol 2014;9:403-9.

125.Padda SK, Riess JW, Schwartz EJ, et al. Diffuse high intensity PD-L1 staining in thymic epithelial tumors. J Thorac Oncol 2015;10:500-8.

126. Yokoyama S, Miyoshi H, Nishi T, et al. Clinicopathologic and Prognostic Implications of Programmed Death Ligand 1 Expression in Thymoma. Ann. Thorac. Surg 2016;101:1361-9.

127. Hassan R, Thomas A, Patel MR, et al. Avelumab (MSB0010718C; anti-PD-L1) in patients with advanced unresectable mesothelioma from the JAVELIN solid tumor phase Ib trial: Safety, clinical activity, and PD-L1 expression. J Clin Oncol 2016;34:abstr 8503. 